Extended Abstract

\title{
Music and the Modeling Approach to Genetic Systems of Biological Resonances
}

\author{
Sergey Petoukhov \\ Institute of Machines Studies, Russian Academy of Sciences, Moscow, 101990, Malyi Kharitonievskiy \\ pereulok, 4 . \\ E-Mail: spetoukhov@gmail.com
}

Tel.: +7-915-092-8565

Accepted:

\section{Introduction}

Any living organism is a great chorus of agreed oscillatory processes, which are connected with their genetic inheritance along chains of generations. Since ancient times, chronomedicine believes that all diseases are the result of disturbances in the ordered set of oscillatory processes. From a formal point of view, a living organism is an oscillating system with a large number of degrees of freedom. Resonances in such a system can serve as mechanisms for harmonization and ordering of its set of oscillatory processes. The possibility of resonance approvals has long attracted the attention of many researchers. The presentation represents the author's conception of resonant genomes.

\section{Methods of modeling}

All natural objects - both living and others - have resonant properties. Whether there is a specifity of biological resonant properties, which are inherited genetically? The presentation shows the author's model approach, the results of which give evidences in favor of the specificity of the biological system of inherited resonances. This model approach is based on a relatively narrow class of systems of resonances, which is associated with the eigenvalues and eigenvectors of $\left(2^{\mathrm{n}} * 2^{\mathrm{n}}\right)$-matrices of tensor families based on the tensor product of $(2 * 2)$-matrices.

Matrices are endowed with a remarkable property of displaying resonances. Physical phenomenon of resonances is familiar to everyone. The passage of the signal "s" through the acoustic system $\mathrm{A}$, which is represented by the matrix $\mathrm{A}$, is modeled by the expression $\mathrm{y}=\mathrm{A} * \mathrm{~s}$. If the input signal "s" is a resonant tone, then the output signal " $y$ " repeats it up to a scale factor $y=\lambda *$ s. In the 
matrix the quantity of resonant tones corresponds to its size and the quantity of freedom degrees of the system, which it represents. In vibration theory these resonant tones are called eigenvectors of the matrix, and the scale factors $\lambda_{i}$ are called its eigenvalues, a set of which is a spectrum of the system A (or the matrix $\mathrm{A}$ ). Frequencies $\omega_{\mathrm{i}}=\lambda_{\mathrm{i}}^{0.5}$ are called the natural frequencies of the system, and the corresponding eigenvectors are called its own forms of oscillations. These free undamped oscillations occur in the system in the absence of friction forces in it. Free oscillations of the system determine its behavior in many other conditions.

This report examines the spectra of $\left(2^{\mathrm{n}} * 2^{\mathrm{n}}\right)$-matrices, which are generated by tensor products of the original $(2 * 2)$ matrices and which are used to model some genetic phenomena and structures. The tensor product of matrices has long been used in mathematics, computer science, management theory, coding theory, physics, etc. The tensor product of matrices, which correspond to initial oscillatory systems, allows you to jump to matrices of systems of the increased quantity of freedom degrees.

\section{Results and Discussion}

Tensor products of matrices have the important property of "inheritance" of eigenvalues of original matrices: if original matrices $\mathrm{V}$ and $\mathrm{W}$ have their eigenvalues $\lambda_{\mathrm{i}}$ and $\mu_{\mathrm{j}}$ respectively, then all the eigenvalues of their tensor product are equal to $\lambda_{\mathrm{i}}{ }^{*} \mu_{\mathrm{j}}$. Features of such inheritance of eigenvalues $\left(\lambda_{\mathrm{i}}, \mu_{\mathrm{j}}, \ldots\right)$ of original matrices in cases of tensor multiplication of the matrices can be conveniently represented in the form of "tables of inheritance of eigenvalues of the matrices". The author reveals that these mathematical "tables of inheritance" coincide structurally with phenomenological Punnet squares, which are widely used in the field of genetics from 1906 year to describe poly-hybrid crosses of organisms in accordance with Mendel's laws. But in the case of Punnet squares, alleles of genes are used, not the eigenvalues of matrices. This coincidence generates the following idea:

- Alleles of genes and their combinations can be interpreted as the eigenvalues of $\left(2^{\mathrm{n} *} 2^{\mathrm{n}}\right)$ matrices from the tensor families of matrices of oscillatory systems. This model approach focuses on the possible importance for genetic systems a special class of mutually related resonances from tensor families of matrices, which play the role of biological "matrix archetypes."

Science has led to a new understanding of life itself: "Life is a partnership between genes and mathematics" [1]. But what kind of mathematics can be a partner for the genetic coding system? The author shows some additional evidences that mathematics of eigenvalues and eigenvectors of matrices from the tensor families of matrices (that is mathematics of special systems of resonances) is also useful to model phenomenological structures of alphabets of DNA: 4 nitrogenous bases (adenin A, cytosine $\mathrm{C}$, guanine $\mathrm{G}$, thymine $\mathrm{T}$ ); 16 duplets, 64 triplets. For example, this model approach reveals hidden connections of the genetic alphabets with Walsh functions and Hadamard matrices, which are widely used in information technologies of noise-immune coding. Resonances can be carriers of information. Our set of such results gives evidences in favor of the following:

- Genetic alphabets are systems of resonances; respectively, the genetic code is the code of systems of resonances; genetic texts on the basis of these alphabets are texts on a language of resonances.

From a formal point of view, our vocal apparatus is an oscillatory system with many freedom degrees. In his oral speech and singing, a person utilizes his innate ability to use acoustic resonances, reproduce sounds of speech and music with specific spectral composition, and use the resonances as carriers of information. According to the classics of structural linguistics (R. Jakobson et al.), our 
linguistic language did not come out of nowhere, but it is an extension and superstructure of the genetic language, which is the oldest among all languages. In light of this idea, our discovery of the deep connection of genetic structures with mathematical structures of resonances looks natural. The theory of resonant genomes provides also models of psychophysical law of Weber-Fechner and phyllotaxis laws of morphogenesis in terms of the eigenvalues of matrices of oscillatory systems. These researches about matrix genetics of inherited systems of biological resonances are a continuation of our previous works in the field of matrix genetics [2-4].

Taking into account the musical aspect of our researches, a new slogan can be proposed: a living body is a musical instrument (a synthesizer with an abundance of rearrangements resonant modes). Music is a game in systems of acoustic resonances, to which a person is very predisposed, though he does not have a specialized body of music perception, perceiving music by means of the whole being. During tens of thousands of years, he creates musical instruments, adjusting them to specific systems of resonances, which lead to appearance of his emotions, formication and tears. Repeating genetic processes of agreed complications of living bodies, people over the centuries have learned to combine individual instruments and singers into the orchestras and choirs as oscillatory systems with increased number of freedom degrees. The study of the evolution of musical culture during different historical ages may be important, including, for the birth of heuristic associations to develop theories and methods of research in genetics and bioinformatics.

\section{Conclusions}

The conception of resonant genomes links music and genetic systems. It is based on the theory of resonances of vibrational systems with many freedom degrees. It's able to contribute to the development of musical culture and its tools, and provide some new approaches to music therapy, popular around the world.

Oscillatory processes not only accompany the functioning of the organism, being physiologically necessary, but they are also widely used in physical therapy and medical diagnostics. Vibrations from outside world are the cause of the occupational disease - vibration disease. It is obvious that increased knowledge in the field of genetic science, based on the biological application of methods of mathematical and engineering informatics, could have a positive impact on the development of new methods and means to address many of problems of modern society.

\section{References}

1. Stewart I. Life's other secret: The new mathematics of the living world. New-York: Penguin, 1999.

2. Petoukhov S.V. Matrix genetics, algebras of the genetic code, noise-immunity. RCD: Moscow, Russia, 2008, 316 p. (in Russian, http://petoukhov.com/)

3. Petoukhov S.V., He M. Symmetrical Analysis Techniques for Genetic Systems and Bioinformatics: Advanced Patterns and Applications. IGI Global: Hershey, USA 2010, 271 p.

4. Darvas G., Koblyakov A., Petoukhov S., Stepanyan I. Symmetries in molecular-genetic systems and musical harmony. Symmetry: Culture and Science, 2012, vol. 23, № 3-4, p. 343-375.

(C) 2015 by the authors; licensee MDPI and ISIS. This abstract is distributed under the terms and conditions of the Creative Commons Attribution license. 